\title{
Umbilical cord sclerosis as an indicator of congenital syphilis
}

\author{
S KNOWLES, TERESA FROST \\ From the Queen Victoria Hospital, Rose Park, South Australia
}

SUMMARY In two cases of congenital syphilis examination of the umbilical cord showed a distinctive picture of perivascular sclerosis and inflammation. Large numbers of spirochaetes were shown in the sclerotic zone in both cases. No organisms were demonstrable in the placental disc and there were no morphological changes suggestive of syphilis. It is concluded that the finding of sclerosing funisitis, together with the clinical circumstances, should raise the possibility of congenital syphilis.

The examination of the umbilical cord is a hallowed tradition in midwifery which is often fruitless but may lead to the detection of a single umbilical artery, a true knot, or other gross abnormality. Microscopical evidence of umbilical cord inflammation is now usually thought to be associated with chorionamnionitis but at the turn of the century, umbilical cord inflammation was interpreted as pathognomonic of congenital syphilis.' Subsequent studies set out to establish that omphalitis or funisitis was not always, or indeed often, caused by syphilis. ${ }^{23}$ This was so successfully shown that Treponema pallidum is now rarely considered in the differential diagnosis of inflammation of the placental disc or its associated tissues. We report one case in which the examination of the umbilical cord led to the diagnosis of syphilis in an apparently normal infant and another in which it confirmed the diagnosis of congenital syphilis.

\section{Case reports}

\section{CASE 1}

A 17 year old aboriginal girl had delivered an infant at the age of 16 who died of congenital syphilis in the late neonatal period. She was treated at that time for active syphilis but was lost to follow up until she presented during her next pregnancy at about 16 weeks' gestation. The pregnancy was complicated by vaginal candidiasis, scabies, and persistent urinary tract infection. Repeat serum tests for syphilis showed a four-fold rise in Rapid Plasma Reagin titre from 1/16 to $1 / 64$ by the 32 nd week, indicating active maternal infection. In view of the serological results the mother was treated with penicillin in one $1.8 \mathrm{~g}$ dose. She went into spontaneous premature labour seven days later and was delivered of a liveborn girl weighing $2280 \mathrm{~g}$.

The infant had apgar scores of 9 at one minute and 10 at five minutes after delivery and showed no external stigmata of congenital syphilis. At delivery the placenta was thought to be healthy but, in view of the maternal history, it was sent for pathological examination. Macroscopical examination of the vellamentous cord showed a single umbilical artery. The $10 \mathrm{~cm}$ of cord adjacent to the insertion into the placental membranes showed conspicuous sclerosis (fig 1). The placental disc itself was unremarkable.

Histological examination of the sclerosed length of umbilical cord showed a dense band of debris surrounding both vessels which included degenerating inflammatory cells. No well preserved inflammatory cells were present in the muscular coat of either the vein or artery. Warthin-Starry staining of the cord showed many well preserved spirochaetes, mostly localised within the sclerotic zone (fig 2). No organisms were shown within the placental disc despite a careful search.

Cord blood from the infant showed positive Treponema pallidum haemagglutination and weakly reactive fluorescent treponemal antibody absorption. IgM capture enzyme immunoassay was non-reactive. These findings were regarded as consistent with treponemal infection in late pregnancy. The infant was kept under close observation in the neonatal nursery and was treated with penicillin. During this time she developed radiological evidence of an indeterminate pneumonitis which was thought to be consistent with congenital syphilis. There were also minor bone changes suggestive of very early syphilitic infection. At no time was the infant symptomatic, hepatospleno- 


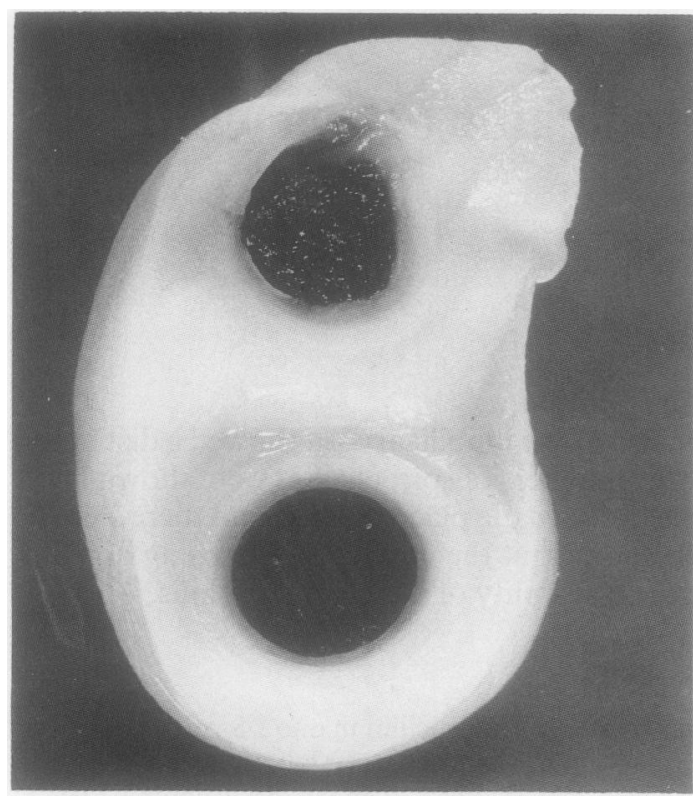

Fig 1 Transverse section of the umbilical cord showing single umbilical artery and striking sclerosing funisitis. Both vessels are surrounded by firm pale rings of sclerosis which were visible through Wharton's jelly on external examination of the card.

megaly was never a feature, and studies of the cerebrospinal fluid yielded quite normal results. She was discharged into the care of her mother at 33 days of age.

\section{CASE 2}

A 26 year old aboriginal woman with five previous pregnancies, three with successful outcomes and two which had resulted in spontaneous miscarriage, had a history of syphilis which had been treated, apparently successfully, three years before the index pregnancy. She was admitted in preterm labour at 32 weeks' gestation with a cervix dilated to $7 \mathrm{~cm}$. Delivery resulted in a liveborn infant with apgar scores of 4 and 6 who deteriorated rapidly and died within 24 hours with clinical and radiological evidence of congenital infection. Rigorous examination of multiple blocks of fetal tissue failed to show the presence of spirochaetes. Retrospective examination of blocks taken from the umbilical cord at the time of delivery, however, showed a picture of sclerosing funisitis resembling that seen in fig 1. Warthin-Starry staining of the blocks showed a profusion of spirochaetes within the areas of sclerosis.

\section{Discussion}

Congenital syphilis is a preventable, potentially fatal

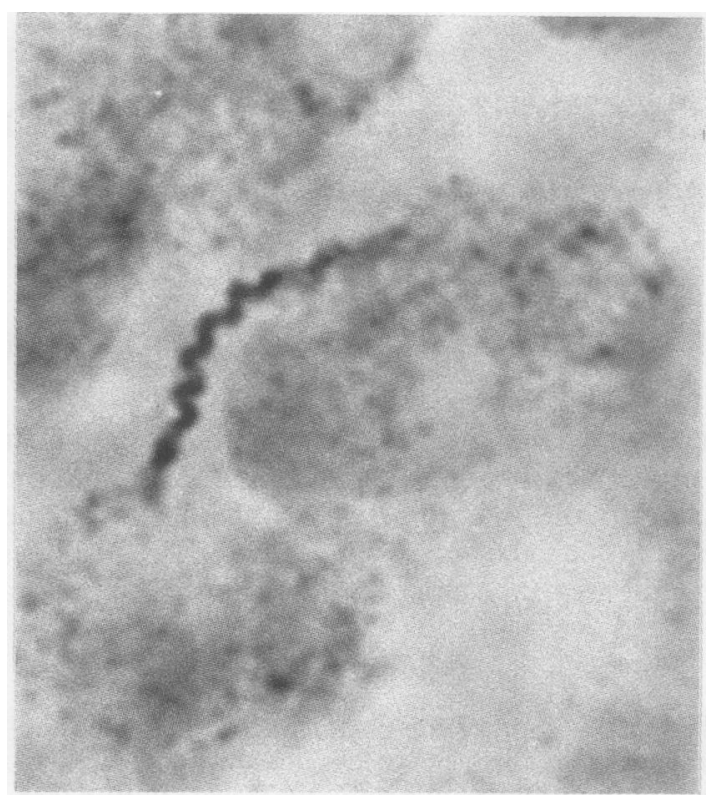

Fig 2 Histological examination of necrotic tissue in areas of sclerosis showing numerous spirochaetes despite administration of prenatal antibiotics (Warthin-Starry stain.)

infection which remains a problem in all countries including those of the first world. ${ }^{4}$ The fact that the pathological examination of the placenta has been eclipsed by serological investigation as an aid to the diagnosis of congenital syphilis has already been pointed out by Russell and Altschuler. ${ }^{5}$ In their description of three cases these authors list a triad of features shown by the syphilitic placenta-focal villitis, endovascular and perivascular proliferation, and relative villous immaturity. They do not mention umbilical lesions. Unlike their cases, we have failed to show the presence of spirochaetes within the placental disc. Focal villous oedema was present but, although some of the villous vessels showed a modest degree of muscular proliferation, this was not accompanied by any form of inflammatory infiltrate. These changes would have been very unlikely to have attracted undue attention from the pathologist under ordinary circumstances.

Routine examination of the umbilical cord, either by frozen section ${ }^{6}$ or after standard processing ${ }^{7}$ has been explored as a means of identifying those infants at greatest risk from perinatal infection but with only limited success. Interestingly, both cases of confirmed syphilis that were described in the latter study had normal umbilical cords.

Sclerosing funisitis is much less common than the acute inflammatory changes seen in the cord in association with chorionamnionitis and, although it 
may be seen in association with ascending infections, ${ }^{8}$ it has also been reported as an isolated finding. ${ }^{9}$ In a series referred to by deSa, ${ }^{10}$ candidal and unspecified bacterial infections are listed as causes of this very recognisable condition. He does not refer to spirochaetal infection but does point out that in most of the cases where no causative organism has been found, anaerobic culture and cultures for mycoplasma and chlamydia were not undertaken.

We suggest that, in suitable clinical circumstances, a Warthin-Starry stain may also be of value in elucidating the cause of sclerosing funisitis and may be beneficial to the subsequent management of the infant.

We thank Drs Keith Lovell and Helen Chambers for their kind permission to report these cases.

\section{References}

1 Bondi J. Die syphilitischen Veraenderungen der Nabelschnur. Arch f Gynak 1903;1xix:223-48.
2 Creadick AN. The frequency and significance of omphalitis. Surg Gynecol Obstet 1920;30:278-83.

3 Siddall RS. The significance of inflammation of the umbilical cord. Am J Obstet Gynecol 1927;14:192-6.

4 Ewing CI, Roberts C, Davidson DC, Arya OP. Early congenital syphilis still occurs. Arch Dis Child 1985;60:1128-33.

5 Russell P, Altschuler G. Placental abnormalities of congenital syphilis. A neglected aid to diagnosis. Am J Dis Child 1974;128:160-3.

6 Wilson MG, Armstrong DH. Inflammation of the umbilical cord and neonatal illness. Am J Obstet Gynecol 1964;7:843-9.

7 Overbach AM, Daniel SJ, Cassady G. The value of umbilical cord histology in the management of potential perinatal infection. $J$ Pediatr 1970;76:22-31.

8 Bourne GL. The Human amnion and chorion. London: LloydLuke, 1962.

9 Malpas P. A case of constrictive sclerosis of Wharton's jelly associated with detachment of the placenta. Journal of Obstetrics and Gynaecology of the British Commonwealth 1968; 75:678-9.

10 deSa DJ. Diseases of the umbilical cord. In: Perrin E, ed. Pathology of the placenta. New York: Churchill Livingstone, 1984:121-39.

Requests for reprints to Dr S Knowles, Laboratory Services, King Edward Memorial Hospital for Women, Sublaio, Western Australia 6008. 